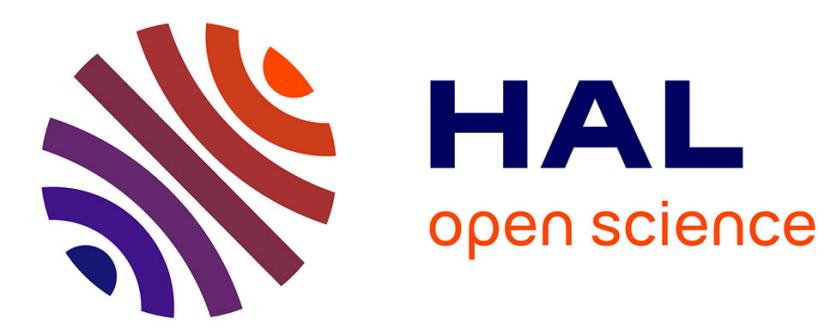

\title{
Long range passive RADAR interrogation of subsurface acoustic passive wireless sensors using terrestrial television signals
}

\author{
Melvin Paquit, Lilia Arapan, Weike Feng, Jean-Michel Friedt
}

\section{- To cite this version:}

Melvin Paquit, Lilia Arapan, Weike Feng, Jean-Michel Friedt. Long range passive RADAR interrogation of subsurface acoustic passive wireless sensors using terrestrial television signals. IEEE Sensors Journal, 2020, pp.5. hal-02993770

\section{HAL Id: hal-02993770 \\ https://hal.science/hal-02993770}

Submitted on 7 Nov 2020

HAL is a multi-disciplinary open access archive for the deposit and dissemination of scientific research documents, whether they are published or not. The documents may come from teaching and research institutions in France or abroad, or from public or private research centers.
L'archive ouverte pluridisciplinaire HAL, est destinée au dépôt et à la diffusion de documents scientifiques de niveau recherche, publiés ou non, émanant des établissements d'enseignement et de recherche français ou étrangers, des laboratoires publics ou privés. 


\title{
Long range passive RADAR interrogation of subsurface acoustic passive wireless sensors using terrestrial television signals
}

\author{
Melvin Paquit, Lilia Arapan, Weike Feng, Jean-Michel Friedt
}

\begin{abstract}
Index Terms-passive RADAR, passive sensor, Surface Acoustic Wave, wireless

Abstract-Acoustic reflective delay lines are ideally suited as cooperative targets for passive wireless sensing: a dedicated temperature sensor was designed and manufactured to demonstrate passive bi-static measurement using $25 \mathrm{~kW}$ terrestrial video broadcast emission at a range of $4850 \mathrm{~m}$, with a continuous refresh rate of a measurement every second. The sensor was buried $5 \mathrm{~cm}$ deep in sand with a reader antenna located $10 \mathrm{~cm}$ from the sensor, a deployment scenario representative of distributed passive sensors for civil engineering monitoring.
\end{abstract}

\section{INTRODUCTION}

Passive RADAR is well known for its stealth and not requiring the licences needed to emit high power target illumination signal [1], [2]. Passive cooperative targets are well known for their ability to act as sensors interrogated wirelessly through the analysis of their RADAR cross-section, whether illuminated by cooperative [3], non-cooperative [4] or noise-like sources [5], [4]. Unlike Radio Frequency IDentifiers (RFID) or electromagnetic energy harvesters, Surface Acoustic Wave (SAW) transducers based on the linear time-variant conversion of an electromagnetic signal to an acoustic wave by a piezoelectric substrate do not require reaching the diode threshold voltage for the rectifier operating the microcontroller to operate [6]. We have demonstrated passive cooperative target measurement using passive RADAR using a WiFi interrogation signal [7], [8]. While the broadband signal is widely available in urban environments, the measurement range is limited by the emitted power. Furthermore, the microwave signal hardly penetrates dielectrics, especially if wet with moisture.

Here we consider the well known broadband powerful emitter available in most urban areas: Digital Video Broadcast Terrestrial (DVB-T) emitters. We design a dedicated SAW cooperative target for sub-surface temperature sensing using one of the available DVB-T channels. The lower frequency DVB-T signal (with respect to $\mathrm{WiFi}$ ) penetrates soil and allows

Sensors were manufactured in the FEMTO-ST cleanroom facility MIMENTO (Besançon, France). V. Soumann (FEMTO-ST) mapped the sensor responses at the wafer level and packaged the devices.

J.-M. Friedt is associate professor at Franche-Comté University with his research activities hosted by the Time \& Frequency department of the FEMTO-ST Institute in Besançon, France. M.P., L.A. and J.-M. F are with the FEMTO-ST institute, Time \& Frequency department, Besançon, France. W.F is with Xidian University, Xian, China. We acknowledge fruitful discussions with S. Benchabane and D. Rabus (FEMTO-ST) during the review process. E-mail: jmfriedt@femto-st.fr. for a backscattered signal to be measured at long range from the emitter. Such a demonstration opens the path to distributed sensing in urban environments where existing radiofrequency signals are used to collect data on passive sensors distributed in the road, pavement [9] or for sub-surface utility monitoring with no need for dedicated radiofrequency emitter [10].

\section{COOPERATIVE TARGET DESIGN}

Passive cooperative target design aims at introducing some signature in the signal returned by the sensor illuminated by a radiofrequency source. Delaying the signal beyond clutter is the most intuitive approach, with both the slow decay of a high quality factor resonator [11], [12] or the delay of a reflective delay line [13] being widely investigated. In order to match the DVB-T emitter $8 \mathrm{MHz}$-wide broadband signal, the latter approach will be adopted, with time delays in the 1 to $1.5 \mu$ s range selected as a tradeoff between correlation lag and delayed signal loss. Indeed in order to shrink sensor dimensions, the electromagnetic wave $(300 \mathrm{~m} / \mu \mathrm{s}$ velocity) is converted to an acoustic wave (typically $3000 \mathrm{~m} / \mathrm{s}$ ) through the inverse piezoelectric effect of a strongly coupled piezoelectric substrate, in our case $\mathrm{YXI} / 128^{\circ}$ lithium niobate (LNO). The acoustic wave propagates on the surface of the substrate, is reflected by an impedance change when reaching Bragg mirrors patterned on the substrate due to mass and electrical loading since all mirror electrodes are left at floating potential, and returns to the interdigitated transducer (IDT) to be converted back to an electrical (direct piezoelectric effect) signal (Fig. 1).

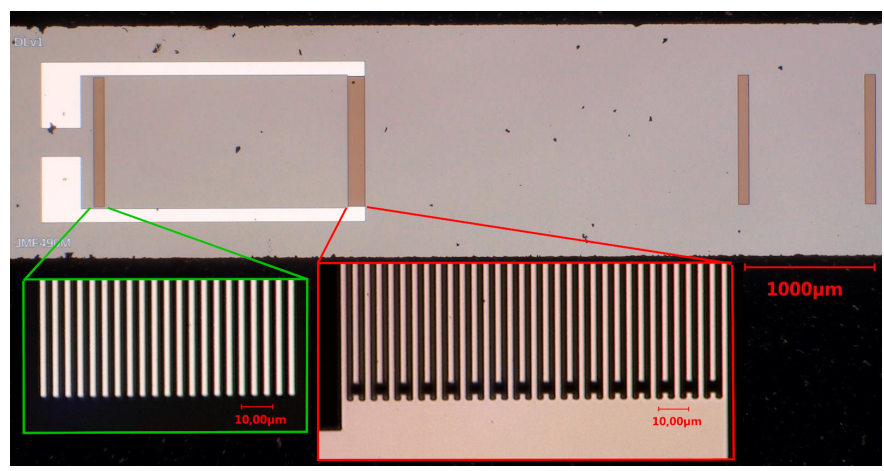

Fig. 1. Layout of the $490 \mathrm{MHz}$ reflective acoustic delay line. Insets are the layout of the IDT and one of the mirrors with floating electrodes. 
The devices used here exhibit return losses [14] in the 13 to $14 \mathrm{~dB}$ range when the interrogation signal spectrum matches the transducer transfer function.
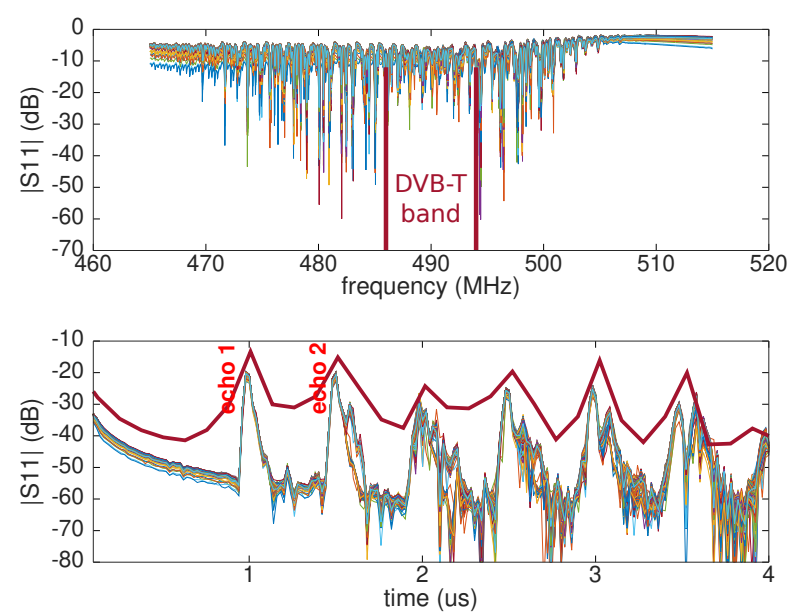

Fig. 2. Frequency domain (top) and time domain (bottom) characteristics of the 419 devices (out of 433 devices patterned on a 4-inch wafer) with the first two echoes (time delays 1.0 and $1.5 \mu \mathrm{s}$ ) exhibiting better than $23 \mathrm{~dB}$ losses on a $50 \mathrm{MHz}$ bandwidth. The thick purple line highlights (top) the $8-\mathrm{MHz}$ wide DVB-T frequency band, and bottom the time-domain response when restricting the frequency range to the DVB-T band, lowering losses to $13 \mathrm{~dB}$ since all electromagnetic power lies in the acoustic transducer bandwidth, at the expenses of poorer time-resolution due to the narrower bandwidth.

Dedicated cooperative targets for passive wireless bistatic measurements have been designed to match two criteria defined by the emitter: center frequency $(490 \mathrm{MHz}$ in our case, or so-called channel 23) and bandwidth $(8 \mathrm{MHz}$ in case of DVB-T). The dedicated design is driven by the relatively narrower bandwidth with respect to the typical $80 \mathrm{MHz}$ wide 2.45 GHz Industrial, Scientific and Medical band in which echoes separated by typical delay intervals of $100 \mathrm{~ns}(10 \mathrm{MHz}$ equivalent bandwidth as the inverse of the echo separation, or less than the available $80 \mathrm{MHz}$ ) are well resolved when illuminated by the broadband signal if e.g. multiple WiFi channels emitted from the same access point are combined. In our case, analyzing a single DVB-T channel emission requires spacing echoes by more than $125 \mathrm{~ns}$ (or $1 /(8 \mathrm{MHz})$ ) to properly separate each contribution. The bandwidth, which can be electrically matched without losses, of an acoustic transducer is determined by its electromechanical coupling coefficient $K^{2}$ : in the case of $\mathrm{YXl} / 128^{\circ} \mathrm{LNO}$, the $K^{2} \simeq 5 \%$ would allow benefiting from a $24.5 \mathrm{MHz}$ broadband signal at $490 \mathrm{MHz}$, but definitely prevents benefiting from adjacent communication channels since the next channel is centered on $514 \mathrm{MHz}$. Typical frequency domain and time-domain characterization of the sensor is given in Fig. 2 emphasizing the excellent yield making such devices cost-effective, with 419 functional chips manufactured on a 4-inch wafer as defined by echo return losses better than $23 \mathrm{~dB}$, out of a total of 433 measured devices. The $23 \mathrm{~dB}$ return loss displayed here is due to the excessively broad frequency range used for exhibiting the whole acoustic device response, leading to excessive losses during the inverse Fourier transform normalization process since a fraction of the incoming energy lying out of the operating frequency band did not propagate as acoustic energy.

The electrodes patterned on the LNO substrates are made of $250 \mathrm{~nm}$-thick sputtered aluminum. The electrode aperture is $1000 \mu \mathrm{m}$ with a mechanical period of $4.03 \mu \mathrm{m}$ for an electrical period of $8.06 \mu \mathrm{m}$, and a metalization ratio of 0.5 . The interdigitated transducer is made of 17 electrode pairs, as expected for optimal coupling as the inverse of $K^{2}$, while the first two mirrors are made of 11 electrode pairs. A tentative additional third mirror is patterned with 11 electrode pairs but will not be used due to excessive losses in the first mirror met in this acoustic path.

\section{COOPERATIVE TARGET INTERROGATION}

The experiment site is located $4850 \mathrm{~m}$ from the $25 \mathrm{~kW}=+74 \mathrm{dBm}$ Montfaucon DVB-T emitter [15] illuminating the Besançon area (France) as shown in Fig. 3: in this setup, the DVB-T covers most of the Besançon downtown area. This signal reaches the cooperative target with a Free Space Propagation Loss - also known as Friis formula [16] $\left(F S P L=20 \log _{10}(d)+20 \log _{10}(f)-147.55 \mathrm{~dB}\right.$ with $f$ the propagated frequency in $\mathrm{Hz}$ received at a range $d$ in meters) of $100 \mathrm{~dB}$, or $-26 \mathrm{dBm}$.

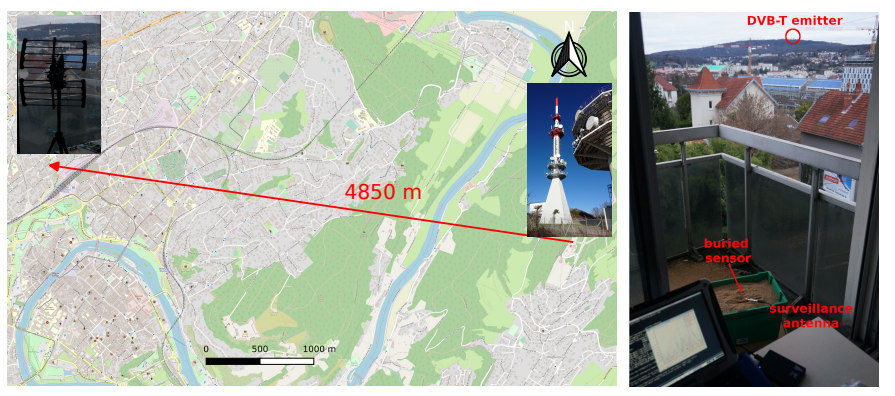

Fig. 3. Left: experiment layout. The Montfaucon DVB-T emitter (inset, right) is located to the east at coordinates (WGS84) $47.242222 \mathrm{~N}, 6.083611 \mathrm{E}$, the sensor and receiver (inset, left) are located $4850 \mathrm{~m}$ to the West of the emitter in direct line of sight. Right: receiver experimental setup.

The sensor acts as a point-like reflector with $13 \mathrm{~dB}$ losses and hence radiates $-39 \mathrm{dBm}$. The receiver located $20 \mathrm{~cm}$ over the sandbox in which the sensor is buried receives a signal attenuated by another $12 \mathrm{~dB}$, assuming all isotropic antenna impedances match: the received signal power is about $-51 \mathrm{dBm}$. Two antennas collect the signal of interest: a 6element Yagi-Uda antenna points directly to the signal from the DVB-T emitter (reference signal) while a dipole is located on the sandbox to collect the signal backscattered by the sensor (surveillance signal). The cross-correlation between these two signals exhibit correlation peaks when the time delay introduced by the cooperative target is detected, with a phase representative of the fine measurement of the acoustic velocity. Indeed the phase of the acoustic wave is $\varphi=2 \pi f \tau$ for a carrier frequency $f$ and a delay $\tau$. Getting rid of the correlated delay sources such as source to target distance requires working on a differential measurement between two echo delays. In our case, two echoes at 1.5 and $1.0 \mu$ s are returned so that $\Delta \tau=0.5 \mu \mathrm{s}$ and the phase rotation is $\Delta \varphi=1540 \mathrm{rad}$. Considering the temperature sensitivity of the piezoelectric 
substrate of $70 \mathrm{ppm} / \mathrm{K}$, the phase sensitivity to temperature is $0.108 \mathrm{rad} / \mathrm{K}$. The time delay introduced by a temperature variation of $100 \mathrm{~K}$ is $70 \mathrm{ppm} / \mathrm{K} \times 100 \mathrm{~K} \times \Delta \tau=3.5 \mathrm{~ns}$. Considering the DVB-T signal bandwidth of $8 \mathrm{MHz}$ and the sampling bandwidth of $12 \mathrm{MHz}$ or a sampling interval of $83.3 \mathrm{~ns}$, such delay will keep the correlation peak within the same bin and only the phase at a fixed delay will be monitored.

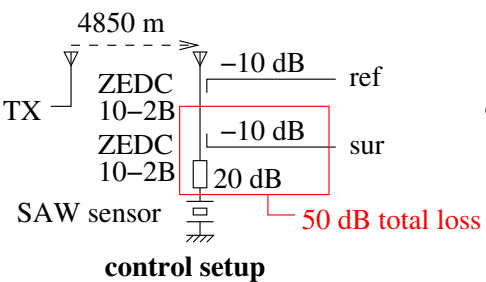

control setup

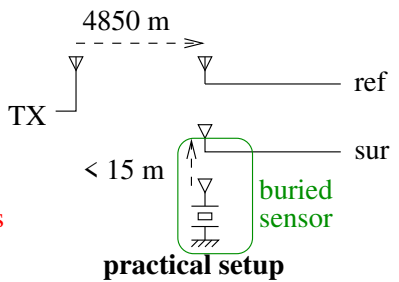

practical setup
Fig. 4. Experimental procedure: initial measurements in a controlled environment involved powering a passive SAW sensors with the attenuated reference signal (ref) collected by a Yagi-Uda antenna (left). Having assessed the attenuation allowing for a usable signal, the bi-directional coupler and attenuators are replaced with a surveillance antenna (sur) and the sensor connected to a dipole antenna is buried in sand for a wireless measurements. "ZEDC-10-2B" refers to the Mini Circuits reference of the directional coupler.

An initial demonstration of the measurement capability uses a single antenna to feed the signal radiated by the DVB-T emitter to a sensor connected to two couplers assembled as a single bi-directional coupler (Fig. 4, left). The outputs of these two couples feed the two coherent channels of an Ettus Research B210 Software Defined Radio receiver sampling at $12 \mathrm{MS} / \mathrm{s}$ (two complex channels). In such a configuration in which two ZEDC-10-2B are connected in a bi-directional coupler configuration (Fig. 5, inset), even a $20 \mathrm{~dB}$ attenuator between coupler and sensor (40 dB two-way attenuation) still allows collecting a signal with better than $6 \mathrm{~dB}$ usable signal to noise ratio (Fig. 5). When measuring a sensor buried in sand, positioning the surveillance antenna close to the sand surface is mandatory to avoid excessive losses at the sandair interface, as is well known from the Ground Penetrating RADAR (GPR) field.

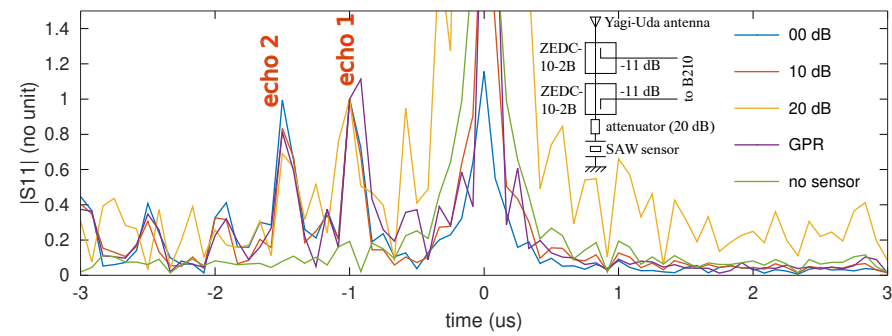

Fig. 5. Passive RADAR response of the SAW sensor: a $10 \mathrm{~dB}$ bidirectional coupler with $0 \mathrm{~dB}, 10 \mathrm{~dB}$ and $20 \mathrm{~dB}$ attenuation between the coupled port and the SAW sensor provides controlled experimental conditions ; a typical wireless GPR measurement at a range of $10 \mathrm{~cm}$ with the sensor buried $5 \mathrm{~cm}$ in sand ; and no sensor. All measurements are normalized to the first echo power, except in the absence of sensor in which the curve is normalized with the wireless measurement power at the first echo location. Inset: experimental setup, in which the reference antenna collects the DVB-T signal and feeds the sensor through an attenuator. See Fig. 4 (left) for a magnified copy of the inset.
Minute acoustic wave delays are measured as the phase of the cross correlation. Indeed the cross correlation xcorr between the reference signal $R$ and the surveillance signal $S$ delayed by a phase $\varphi$ is equal to the phase-delayed crosscorrelation between reference and surveillance signals:

$$
x \operatorname{corr}(R, S \cdot \exp (j \varphi))=\exp (j \varphi) \cdot x \operatorname{corr}(R, S)
$$

Hence, measuring the phase of the correlated signal at the bin of maximum returned power will be representative of the physical quantity affecting the acoustic velocity, in our case temperature (Fig. 6). No free fitting parameter is left in the conversion from phase to temperature in the bottom chart of this figure: the temperature sensitivity $S=70 \mathrm{ppm} / \mathrm{K}$ of $\mathrm{YXI} / 128^{\circ} \mathrm{LNO}$ is tabulated, the echo delay $\tau=1 \mu$ s defined by the sensor design when considering the first reflection echo, and the operating frequency $f=490 \mathrm{MHz}$ by the signal source so that the phase variation $d \varphi$ per unit temperature variation $d T$ is $d \varphi=S \cdot 2 \pi f \tau \times d T \Leftrightarrow d T=d \varphi /(S \cdot 2 \pi f \tau)$
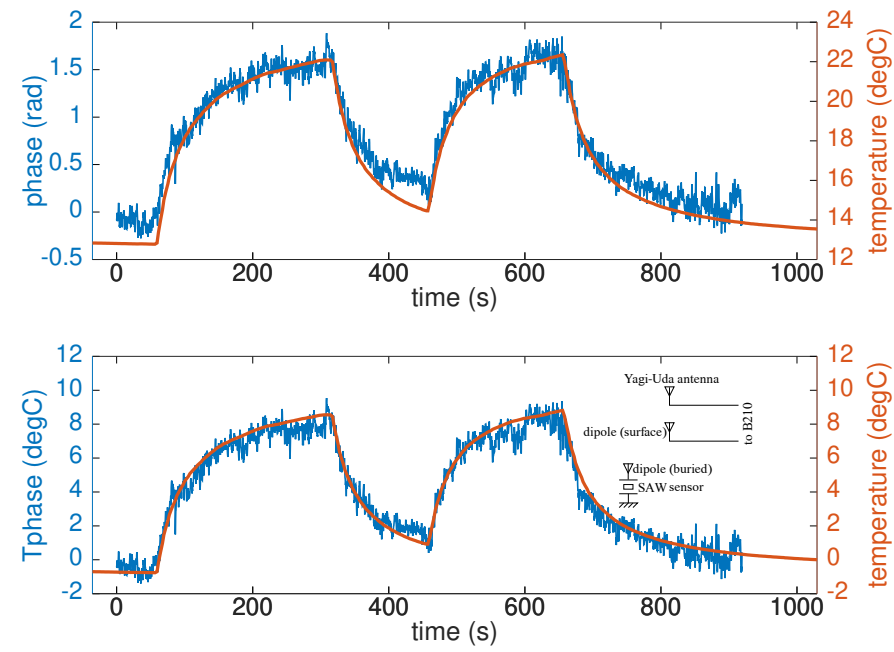

Fig. 6. First echo phase measurement representative of temperature (top), and comparison with a reference temperature measurement thanks to a Pt100 probe glued to the ceramic package holding the sensor. Bottom: conversion from phase to temperature using the tabulated temperature sensitivity constant of the piezoelectric substrate and geometric design quantities. Inset: experimental setup: referring to the inset of Fig. 5, the attenuator is replaced with a wireless link between the sensor and the surveillance antenna connected to the second port of the B210 Software Defined Radio1G receiver. See Fig. 4 (right) for a magnified copy of the inset.

It is worth mentioning that in the cross-correlation xcorr equation searching for matches of delayed copies of the reference signal $R$ in a surveillance signal $S$, the integration length $m \in[1 . . M]$ and lag $n \in[-N: N]$ are two independent quantities:

$$
x \operatorname{corr}(R, S)(n)=\sum_{m=1}^{M} R(m+n) S(m)
$$

where $n$ is defined by the SAW sensor design, in our case echoes delayed by less than $5 \mu$ s or $n \in[ \pm 64]$ since the signals are sampled at $12 \mathrm{MS} / \mathrm{s}$ ( 64 samples $/ 12 \mathrm{MS} / \mathrm{s}=5.3 \mu \mathrm{s})$ while $M$ is defined by the targeted signal to noise ratio. The bin width in the time-domain in which the phase is measured is solely defined by the inverse of the measurement bandwidth, or $83 \mathrm{~ns}$ 
in our case. All our analysis were performed on 1 MS datasets or $83 \mathrm{~ms}$ long measurements. Correlating two signals acts as a sliding averaging operation of the surveillance signal with a weight function given by the reference signal. Thus, the signal to noise ratio improves by correlating as $\sqrt{M}$ and since the bistatic RADAR equation exhibits a link budget dependent on the square of the sensor to receiver distance when the sensor is close to the receiver and far from the emitter (our experimental case), then doubling the sensor-reader measurement range (i.e. multiplying losses by 4 ) requires multiplying $M$ sixteen-fold since $\sqrt{16}=4$.

\section{DISCUSSION: LINK BUDGET AND COMPARISON WITH ELECTROMAGNETIC ENERGY HARVESTERS}

The linear electromechanical conversion process operates whatever incoming power reaches the sensor: the ability to record the backscattered signal is a matter of low enough receiver noise or long enough integration duration in order to lower the noise floor. On the other hand, RFID and electromagnetic energy harvesters [17], [18] require a minimum threshold voltage to power the microcontroller modulating the backscattered signal. While the RFID backscattered modulation is itself decaying as the fourth power of the bistatic range, electromagnetic harvesters store the collected energy and emit back signal only decreasing as the square of the distance. Hence, had the signal been powerful enough to power the energy harvesting system, the global link budget might be more favorable for the latter technique.
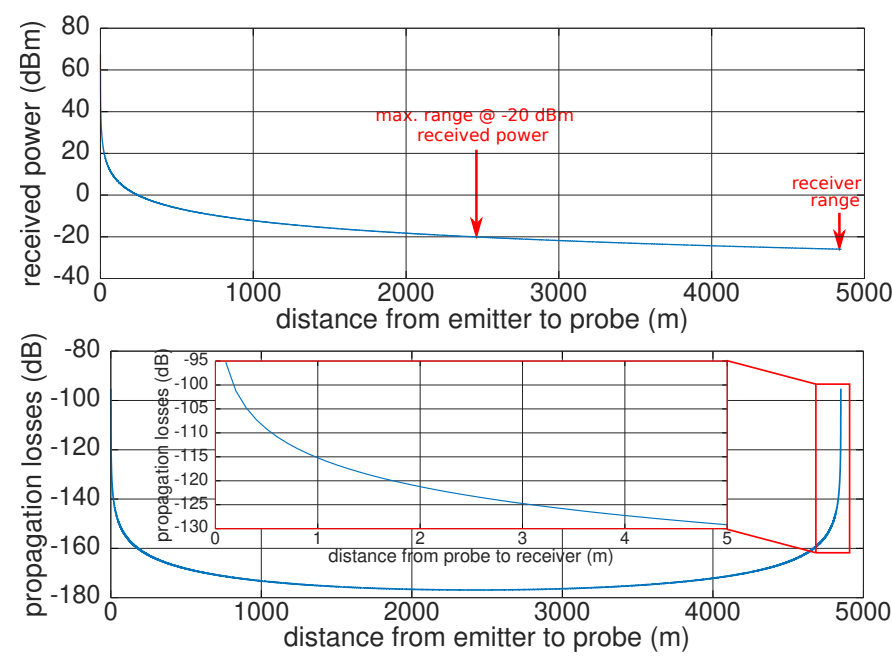

Fig. 7. Top: received power assuming Free Space Propagation losses and isotropic antennas. Bottom: bistatic RADAR link budget assuming isotropic antennas and a sensor located at varying distances along the constant line linking the emitter and receivers.

The tradeoff between powering the sensing system from electromagnetic energy harvesting or monitoring the signal backscattered from an acoustic reflective delay line lies in the range from emitter to sensor and sensor to receiver (Fig. 7). If the receiver power is above threshold, energy harvesting is probably a viable solution, despite a measurement and communication rate limited by the power accumulation until enough energy has been collected for a measurement and transmission.
Considering a threshold power of $-20 \mathrm{dBm}$ below which the rectifier can no longer operate [19], our demonstration of a continuous measurement with a refresh rate below $1 \mathrm{~s}$ would not be possible using electromagnetic energy harvesting and passive sensor backscattering coefficient measurement is the only functional solution. Estimated received power (Fig. 7, top) based solely on Free Space Propagation Loss hints at a range of $2.5 \mathrm{~km}$ at which the received power might reach $20 \mathrm{dBm}$ (emitted power: $+74 \mathrm{dBm}$ at $490 \mathrm{MHz}$ ), definitely out of reach of our SAW sensor located nearly $5 \mathrm{~km}$ away from the emitter (right-most red arrow). At such a range, using the acoustic transducer is the only viable solution, with a receiving antenna necessarily located close to the sensor considering the bi-static link-budget (Fig.7, bottom). This bottom chart indicates the RADAR Free Space Propagation Loss assuming a point-like cooperative target so that the total communication losses along the line of sight is $(4 \pi)^{3} \times d^{2} \times(D-d)^{2} / \lambda^{4}$ with $D=4850 \mathrm{~m}$ the emitter to receiver range, $d$ the sensor distance along this path and $\lambda=c / f=61 \mathrm{~cm}$ the wavelength at $f=490 \mathrm{MHz}$ with $c$ the velocity of light. This latter chart demonstrates why the reader unit must be close to the sensor: the bistatic loss equation, here becoming symmetric by varying the sensor position $d$ along the line of sight and keeping the emitter-receiver distance $D$ constant, hints at sub-100 dB losses, typical of a Ground Penetrating RADAR link budget, at short range. These losses rise to unacceptable levels at longer range as the sensor is located at mid-range between emitter and receiver. Considering that we were able to achieve measurements with better than $6 \mathrm{~dB}$ signal to noise ration with a $20 \mathrm{~dB}$ attenuator $(40 \mathrm{~dB}$ attenuation two-pass loss) connected between sensor and a $10 \mathrm{~dB}$ coupler, such a $50 \mathrm{~dB}$ attenuation of the sensor signal would be achieved with Free Space Propagation Loss at a bistatic range of $15 \mathrm{~m}$ (depth $7.5 \mathrm{~m}$ ) between sensor and receiver antenna (Free Space Propagation Loss computed with $f=490 \cdot 10^{6}$ and $d=15$ ), assuming all other conditions being ideal (no electromagnetic losses in soil and matched antennas) since we keep the transmitter to reference antenna range constant when replacing the coupler and attenuator with a separate surveillance antenna probing the sensor signal. Due to electromagnetic losses as the radiofrequency wave propagates in soil in addition to the poor coupling of the electromagnetic wave propagating in air when penetrating soil at grazing angle, an interrogation range of a meter at best is expected: indeed (Fig. 8), using the Fresnel electromagnetic propagation equations at a planar interface, the coupling losses at grazing angle are as high as $15 \mathrm{~dB}$. In Fig. 8, the TE-polarized ( $s$-polarized) DVB-T signal reaches the ground with relative permittivity ranging from 2 (dry sand) to 80 (water) and the transmitted power is computed as unity minus the reflected power $R_{s}=\left(\frac{n_{1} \cos t_{i}-n_{2} \cos t_{t}}{n_{1} \cos t_{i}+n_{2} \cos t_{t}}\right)^{2}$ where $n_{1} \sin t_{i}=n_{2} \sin t_{t}$. Since he sensor acts as a point like source and radiates a sphere centered on its position, the receiver $\mathrm{RX}$ closely coupled to soil will not be affected by this transmission loss wherever it is located at the ground-air interface. In our case, the Montfaucon DVB-T emitter is located at an altitude of $605 \mathrm{~m}$ while the mean downtown altitude of Beançon (France) is $260 \mathrm{~m}$, or an incidence angle $t_{i} \simeq 85^{\circ}$ as indicated 
by the green vertical line, yielding transmission losses in the $10 \mathrm{~dB}$ range for wet sand with permittivity in the 4 to 10 range. With this additional loss source, the bi-static range drops to $5 \mathrm{~m}$ (2.5 m depth), excluding impedance mismatch of the sensor antenna and the electromagnetic propagation losses in conducting soil.
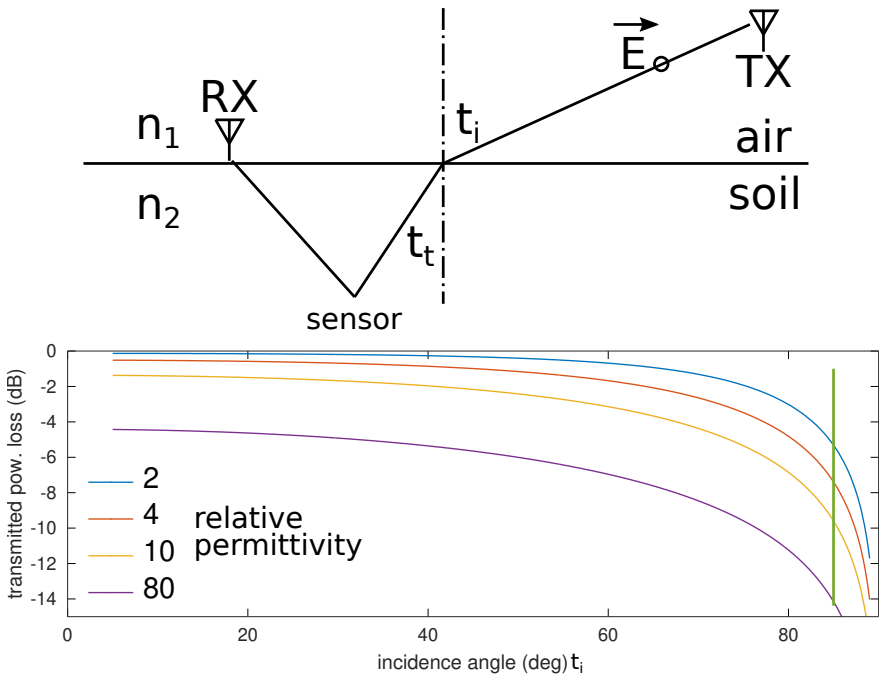

Fig. 8. Transmission loss as a function of incoming angle $t_{i}$ of the electromagnetic wave emitted by the transmitter TX, reaching the air-ground interface before reaching the buried sensor which acts as a point like source and radiates back towards the receiver RX assumed to be closely bound to the soil. The optical index $n$ is related to the relative permittivity $\varepsilon$ by $n=\sqrt{\varepsilon}$ indicated in the legend of the chart. Top: simulation geometry.

When stating earlier that correlation integration time could be increased to improve measurement range - doubling $d$ by multiplying by sixteen $M$ under the assumption that $D \gg d$, we assumed the linear behavior of the sensor with incoming power, which is not valid for energy harvesting systems using a rectifier bridge to control silicon-based integrated circuits. The limit to increasing integration time - beyond practical usage measurement refresh rates - lies in the software defined radio implementation of the receiver: when the surveillance signal becomes so weak that stochastic processes no longer induce least significant bit fluctuations in the noise measurement, quantization of the analog to digital converter will limit the digital pulse compression capability signal to noise ratio improvement during digital signal processing.

\section{CONCLUSION}

A surface acoustic wave reflective delay line was designed to act as cooperative target for passive wireless sensing using ambient electromagnetic energy using passive bistatic RADAR measurement techniques. We demonstrate continuous $(1 \mathrm{~Hz}$ refresh rate) measurement of temperature by a sensor located $5 \mathrm{~cm}$ deep in sand with a collecting antenna located $10 \mathrm{~cm}$ from the sensor, both parts of the experimental setup located $4850 \mathrm{~m}$ from the terrestrial digital video broadcast $25 \mathrm{~kW}$ emitter. We compare this link budget with electromagnetic energy harvesters published in the literature and argue that such a measurement would not be possible with silicon based measurement systems, hence demonstrating in this context the superiority of the linear piezoelectric substrate.

\section{REFERENCES}

[1] H. D. Griffiths and C. J. Baker, An introduction to passive radar. Artech House, 2017.

[2] H. Kuschel, D. Cristallini, and K. E. Olsen, "Tutorial: Passive radar tutorial," IEEE Aerospace and Electronic Systems Magazine, vol. 34, no. 2, pp. 2-19, 2019.

[3] F. Lurz, T. Ostertag, B. Scheiner, R. Weigel, and A. Koelpin, "Reader architectures for wireless surface acoustic wave sensors," Sensors, vol. 18, no. 6, p. 1734, 2018.

[4] W. Feng, J.-M. Friedt, G. Goavec-Merou, and M. Sato, "Passive radar delay and angle of arrival measurements of multiple acoustic delay lines used as passive sensors," IEEE Sensors Journal, vol. 19, no. 2, pp. 594602, 2018.

[5] J. R. Humphries, F. K. Reed, C. P. Carmichael, P. L. Fuhr, T. J. McIntyre, A. R. Weeks, and D. C. Malocha, "Noise radar approach for interrogating saw sensors using software defined radio," IEEE Sensors Journal, vol. 17, no. 20, pp. 6760-6769, 2017.

[6] C. Campbell, Surface Acoustic Wave Devices for Mobile and Wireless Communications, Four-Volume Set. Academic press, 1998.

[7] J.-M. Friedt, G. Goavec-Merou, G. Martin, W. Feng, and M. Sato, "Passive radar acoustic delay line sensor measurement: demonstration using a WiFi (2.4 GHz) emitter and WAIC-band (4.3 GHz)," in 2018 6th IEEE International Conference on Wireless for Space and Extreme Environments (WiSEE). IEEE, 2018, pp. 54-61.

[8] W. Feng, J.-M. Friedt, G. Goavec-Merou, and M. Sato, "Passive radar delay and angle of arrival measurements of multiple acoustic delay lines used as passive sensors," IEEE Sensors Journal, vol. 19, no. 2, pp. 594 602, 2018.

[9] F. Orfei, C. B. Mezzetti, and F. Cottone, "Vibrations powered LoRa sensor: An electromechanical energy harvester working on a real bridge," in 2016 IEEE SENSORS. IEEE, 2016, pp. 1-3.

[10] K. M. Farinholt, G. Park, and C. R. Farrar, "RF energy transmission for a low-power wireless impedance sensor node," IEEE Sensors Journal, vol. 9, no. 7, pp. 793-800, 2009.

[11] F. Seifert, A. Pohl, R. Steindl, L. Reindl, M. J. Vellekoop, and B. Jakoby, "Wirelessly interrogable acoustic sensors," in Proceedings of the 1999 Joint Meeting of the European Frequency and Time Forum and the IEEE International Frequency Control Symposium (Cat. No. 99CH36313), vol. 2. IEEE, 1999, pp. 1013-1018.

[12] W.-E. Bulst, G. Fischerauer, and L. Reindl, "State of the art in wireless sensing with surface acoustic waves," IEEE Transactions on Industrial Electronics, vol. 48, no. 2, pp. 265-271, 2001

[13] L. Reindl, G. Scholl, T. Ostertag, C. Ruppel, W.-E. Bulst, and F. Seifert, "SAW devices as wireless passive sensors," in 1996 IEEE Ultrasonics Symposium. Proceedings, vol. 1. IEEE, 1996, pp. 363-367.

[14] K.-Y. Hashimoto, Surface acoustic wave devices in telecommunications. Springer, 2000.

[15] Information dated March 3rd, 2014 collected on https://www.csa.fr/ csagabarit/liste/plan/pass, the French Superior Council of the Audiovisual agency, in December 2019.

[16] H.T.Friis, "A note on a simple transmission formula," Proc. I.R.E., pp. 254-256, 1946.

[17] H. Nishimoto, Y. Kawahara, and T. Asami, "Prototype implementation of wireless sensor network using TV broadcast RF energy harvesting," in Proceedings of the 12th ACM international conference adjunct papers on Ubiquitous computing-Adjunct. ACM, 2010, pp. 373-374.

[18] R. Vyas, H. Nishimoto, M. Tentzeris, Y. Kawahara, and T. Asami, "A battery-less, energy harvesting device for long range scavenging of wireless power from terrestrial TV broadcasts," in 2012 IEEE/MTT-S International Microwave Symposium Digest. IEEE, 2012, pp. 1-3.

[19] Y. Luo, L. Pu, G. Wang, and Y. Zhao, "RF energy harvesting wireless communications: RF environment, device hardware and practical issues," Sensors, vol. 19, p. 3010, 2019. 